\title{
Front Matter: Volume 11285
}

, "Front Matter: Volume 11285," Proc. SPIE 11285, Silicon Photonics XV, 1128501 (11 March 2020); doi: 10.1117/12.2570190

SPIE. Event: SPIE OPTO, 2020, San Francisco, California, United States 


\section{PROCEEDINGS OF SPIE}

\section{Silicon Photonics XV}

Graham T. Reed

Andrew P. Knights

Editors

3-6 February 2020

San Francisco, California, United States

Sponsored and Published by

SPIE 
The papers in this volume were part of the technical conference cited on the cover and title page. Papers were selected and subject to review by the editors and conference program committee. Some conference presentations may not be available for publication. Additional papers and presentation recordings may be available online in the SPIE Digital Library at SPIEDigitalLibrary.org.

The papers reflect the work and thoughts of the authors and are published herein as submitted. The publisher is not responsible for the validity of the information or for any outcomes resulting from reliance thereon.

Please use the following format to cite material from these proceedings:

Author(s), "Title of Paper," in Silicon Photonics XV, edited by Graham T. Reed, Andrew P. Knights, Proceedings of SPIE Vol. 11285 (SPIE, Bellingham, WA, 2020) Seven-digit Article CID Number.

ISSN: 0277-786X

ISSN: 1996-756X (electronic)

ISBN: 9781510633339

ISBN: 9781510633346 (electronic)

Published by

SPIE

P.O. Box 10, Bellingham, Washington 98227-0010 USA

Telephone +1 3606763290 (Pacific Time) · Fax +1 3606471445

SPIE.org

Copyright (C) 2020, Society of Photo-Optical Instrumentation Engineers.

Copying of material in this book for internal or personal use, or for the internal or personal use of specific clients, beyond the fair use provisions granted by the U.S. Copyright Law is authorized by SPIE subject to payment of copying fees. The Transactional Reporting Service base fee for this volume is $\$ 18.00$ per article (or portion thereof), which should be paid directly to the Copyright Clearance Center (CCC), 222 Rosewood Drive, Danvers, MA 01923. Payment may also be made electronically through CCC Online at copyright.com. Other copying for republication, resale, advertising or promotion, or any form of systematic or multiple reproduction of any material in this book is prohibited except with permission in writing from the publisher. The CCC fee code is 0277 $786 \mathrm{X} / 20 / \$ 18.00$

Printed in the United States of America.

Publication of record for individual papers is online in the SPIE Digital Library.

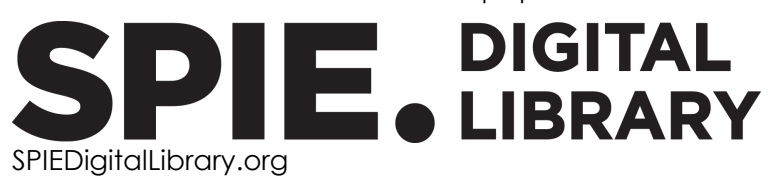

SPIEDigitalLibrary.org

Paper Numbering: Proceedings of SPIE follow an e-First publication model. A unique citation identifier (CID) number is assigned to each article at the time of publication. Utilization of CIDs allows articles to be fully citable as soon as they are published online, and connects the same identifier to all online and print versions of the publication. SPIE uses a seven-digit CID article numbering system structured as follows:

- The first five digits correspond to the SPIE volume number.

- The last two digits indicate publication order within the volume using a Base 36 numbering system employing both numerals and letters. These two-number sets start with 00, 01, 02, 03, 04, 05, 06, 07, 08, 09, OA, OB ... 0Z, followed by 10-1Z, 20-2Z, etc. The CID Number appears on each page of the manuscript. 


\title{
Contents
}

\author{
xii $\quad$ Authors \\ ix Conference Committee
}

PHOTONIC INTEGRATION

1128503 MORPHIC: programmable photonic circuits enabled by silicon photonic MEMS (Invited Paper) [11285-1]

$1128504 \quad$ Epitaxial integration of high-performance quantum-dot lasers on silicon (Invited Paper) [11285-2]

1128506 Multi-chip heterogeneously integrated array of active three-terminal transistor lasers and passive photonic structures for electronic-photonic integration on silicon [1 1285-4]

1128507 Amorphous silicon waveguide escalator: monolithic integration of active components on $3 \mu \mathrm{m}$ SOI platform [1 1285-5]

\section{OPTICAL COMMUNICATIONS I}

$112850 \mathrm{~A} \quad$ Fabrication tolerant high-speed SiP ring modulators and optical add-drop multiplexers for WDM applications [11285-8]

$11285 \mathrm{OB} \quad$ Advanced Si photonics platform for high-speed and energy-efficient optical transceivers for datacom [1 1285-9]

\section{OPTICAL COMMUNICATIONS II}

11285 OC Power-efficient lumped-element meandered silicon Mach-Zehnder modulators [11285-10]

11285 OD Strain induced Pockels effect in silicon for electro-optic modulation [1 1285-11]

11285 OF $16 \times 16$ silicon photonic AWGR for dense wavelength division multiplexing (DWDM) O-band interconnects [11285-13]

$112850 \mathrm{OG}$ Flat-top interleavers based on single MMIs [1 1285-14]

$11285 \mathrm{OH} \quad$ Ultrafast self-induced oscillation in a nonlinear subwavelength grating metamaterial ring resonator [1 1285-15] 


\section{SILICON PHOTONICS ENABLED LIDAR}

11285 0J An overview of silicon photonics for LIDAR (Invited Paper) [1 1285-17]

11285 OK Integrated optical phased arrays: LiDAR, augmented reality, and beyond (Invited Paper) [11285-18]

WAVEGUIDES

11285 OP Suspended subwavelength grating waveguides on SOI for ultra-broadband operation [1 1285-23]

$112850 Q \quad$ Photonic crystal and quasi photonic crystal Ge-on-Si lenses for the combination of QCL array outputs [1 1285-24]

11285 OR Optical reflection from a free carrier-induced front in a slow light waveguide [11285-25]

\section{GE/SI INTEGRATION}

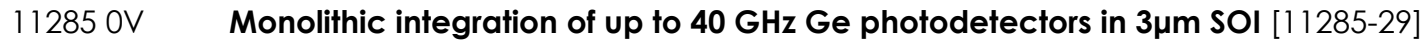

\section{MANUFACTURING TECHNOLOGY}

11285 OY Hot-wire CVD hydrogenated amorphous silicon for multi-layer photonic applications (Invited Paper) [1 1285-32]

1128510 Experimental phase-error extraction and modelling in silicon photonic arrayed waveguide gratings [1 1285-34]

$1128512 \quad$ Electrical annealing for Ge ion-implanted directional couplers [1 1285-36]

OPTICAL DETECTION AND SENSING I

1128513 Micro PA detector: pushing the limits of mid IR photoacoustic spectroscopy integrated on silicon (Invited Paper) [1 1285-37]

$1128515 \quad$ High speed integrated waveguide lateral Si/Ge/Si photodiodes with optimized transit time [1 1285-39]

$1128516 \quad$ Heterodyne detection for the measurement of electro-optical frequency combs generated with a silicon Mach-Zehnder modulator [1 1285-40]

iv 
EMERGING APPLICATIONS II

112851 A Reconfigurable photonic integrated circuits (RPICs) based on functional materials for integrated optical communication applications [1 1285-44]

11285 1C The effect of two-photon absorption on the dynamic range of integrated microwave photonics links [11285-46]

OPTICAL DETECTION AND SENSING II

11285 IG Investigations into group IV photonic waveguides with a wide working optical bandwidth [11285-49]

\section{OPTICAL COMMUNICATIONS III}

$112851 \mathrm{~J} \quad$ Fast thermo-optic optimization of high-order SOI microring optical filters by method of gradient descent [11285-53]

\section{POSTER SESSION}

$112851 \mathrm{~K}$ Design of silicon photonics based accelerometer for displacement sensing applications [11285-54]

11285 IL Low voltage $12 \mathrm{GHz}$ silicon optical electro-absorption modulator (EAM) using a Schottky diode for optical interconnectors in the C-band [1 1285-55]

1128510 Model-based guard ring structure guideline for the enhancement of silicon-based singlephoton avalanche diode characteristics [1 1285-58]

$112851 \mathrm{P} \quad$ A compact structure for realizing electromagnetically induced transparency in a microring resonator [1 1285-59]

11285 IR A widely tunable twin-fano resonator for wavelength monitor [11285-61]

11285 IT Absorptivity enhancement of black silicon using electroless Cu plating [1 1285-63]

$112851 \mathrm{U}$ Laterally asymmetrical photonic crystal waveguide as a compact TM-pass polarization filter for C-band operation [1 1285-64] 
Proc. of SPIE Vol. 11285 1128501-6 Downloaded From: https://www.spiedigitallibrary.org/conference-proceedings-of-spie on 26 Apr 2023
Terms of Use: https://www.spiedigitallibrary.org/terms-of-use 


\section{Authors}

Numbers in the index correspond to the last two digits of the seven-digit citation identifier (CID) article numbering system used in Proceedings of SPIE. The first five digits reflect the volume number. Base 36 numbering is employed for the last two digits and indicates the order of articles within the volume. Numbers start with 00, 01, 02, 03, 04, 05, 06, 07, 08, 09, 0A, 0B...0Z, followed by 10-12, 20-2Z, etc.

Aalto, Timo, 07, 0G, OV

Abasahl, Banafsheh, 03

Abautret, Johan, $0 Q$

Alexoudi, T., OF

Alonso-Ramos, Carlos, OD

Baiocco, Christopher, OK

Baldycheva, Anna, 1A

Barbier, Margaux, 16

Bass, Jake, $1 \mathrm{C}$

Baudot, Charles, 16

Benedikovic, Daniel, OD

Bera, Arijit, 07

Berciano, Mathias, OD

Bernabé, Stéphane, OB, 15

Berthelot, A., 13

Bhargava, Pavan, OK

Boeuf, Frédéric, 16

Bogaerts, Wim, 03, 10

Bourouina, Tarik, IT

Bowers, John E., 04

Bramerie, Laurent, 16

Brea, Brandon, 1C

Brision, Stéphane, OB, 15

Byrd, Matthew J., OK

Cao, Wei, $1 G$

Carlson, John A., 06

Carras, Mathieu, $\mathrm{OQ}$

Cassan, Eric, OD

Chae, Youngcheol, 10

Chen, Bei, IR

Chen, Ray T., $\mathrm{OH}$

Chen, Xia, 12

Cheng, Yue, $\mathrm{OH}$

Cherchi, Matteo, 07, 0G

Chong, Harold M. H., OY

Chung, Chi-jui, $\mathrm{OH}$

Coutard, J. G., 13

Crozat, Paul, OD

Dai, Tingge, $1 R$

Dallesasse, John M., 06

De Heyn, P., OF

Delrosso, Giovanni, OV

Deniel, Lucas, OD, 16

Dominguez Bucio, Thalia, $1 \mathrm{~A}$

Doylend, J. K., OJ

Du, Wei, $1 C$

Duan, Jianan, 04

Dumont, Mario, 04

Dyer, Thomas, OK
Edinger, Pierre, 03

Eich, Manfred, OR

Elsayed, Ahmed A., IT

Errando-Herranz, Carlos, 03

Faneca, Joaquin, IA

Fiers, Martin, 10

Fotiadis, K., OF

Fowler, Daivid, OB

Gaafar, Mahmoud A., OR

Gao, Feng, OV

Garcia Porcel, Marco, 03

Gardes, Frederic Y., IA

Gay, Mathilde, 16

Glière, A., 13

Gossard, Arthur C., 04

Grillot, Frederic, 04

Grosse, P., 15

Guais, Maxime, $0 Q$

Guilhabert, Benoit, $1 G$

Guo, Xiaoqing, 1R

Gupta, S., OJ

Gylfason, Kristinn B., 03

Harjanne, Mikko, OG

Heimala, Päivi, 07, 0V

Herrick, Robert W., 04

Huang, Heming, 04

Hussain, A., IP

Hussein, Magdy, $1 \mathrm{~T}$

Jalas, Dirk, OR

Jeong, Uiseok, $1 \mathrm{~L}$

Jezzini, Moises, 03

Jung, Daehwan, 04

Kapulainen, Markku, OG, OV

Kennedy, M. J., 04

Khaled, Ahmed, IT

Khalil, Diaa, $1 \mathrm{~T}$

Khan, Umar, 03, 10

Khokhar, Ali Z., $1 G$

Kiang, Kian Shen, $1 G$

Kim, Jinsik, 1L

Kim, Kwangwoong, $1 \mathrm{~L}$

Kim, Taehwan, OK

Koshkinbayeva, A., $1 \mathrm{~K}$

Krauss, Thomas F., OR

Lafforgue, Christian, OD

Le Roux, Xavier, OD

Lee, Kyungwoon, $1 \mathrm{~L}$

Lhermet, H., 13

Li, Juntao, OR 
Li, Nanxi, OK

$\mathrm{Li}$, Ting, OP

Liu, Songtao, 04

Magden, Emir Salih, OK

Maisons, Grégory, $O Q$

Malhouitre, Stéphane, OB

Malik, F., IP

Mang, Thomas, OB

Marcaud, Guillaume, OD

Marris-Morini, Delphine, OD, OQ, 16

Marty, Frédéric, IT

Mashanovich, Goran Z., $1 G$

Maximus, A. R., $1 \mathrm{~K}$

Merget, Florian, OA, OC

Michinobu, Tsuyoshi, $\mathrm{OH}$

Milosevic, Milan M., 12

Mitsolidou, C., OF

Mittal, Vinita, OY

Moralis-Pegios, M., OF

Moscoso-Mártir, A., OC

Müller, Juliana, OA

Nedeljkovic, Milos, IG

Nefzaoui, Elyes, $1 T$

Ngo, Huynh, 1J

Niklaus, Frank, 03

Nojić, Jovana, 0A, 0C

Norman, Justin C., 04

Notaros, Jelena, OK

Notaros, Milica, OK

O'Brien, Peter, 03

O'Faolain, Liam, OR

Olivier, Ségolène, $O B$

Oo, Swe Z., OY

Pan, Zeyu, $\mathrm{OH}$

Park, Byungchoul, 10

Park, Hyo-Hoon, $1 \mathrm{~K}$

Park, Jung Ho, $1 \mathrm{~L}$

Peacock, Anna C., OY

Pérez-Galacho, Diego, 16

Petra, Rafidah, OY

Petrov, Alexander YU., OR

Peucheret, Christophe, 16

Pitris, S., OF

Pleros, N., OF

Poulton, Christopher V., OK

Prakash, Chandra, $1 \mathrm{U}$

Qadir, M. Favad, IP

Quack, Niels, 03

Raval, Manan, OK

Reed, Graham T., OY, 12

Ren, Yang, $1 \mathrm{~J}$

Ribaud, Karen, OB

Ribeiro, Antonio, 03

Ruiz-Caridad, Alicia, OD

Sabry, Yasser M., $1 T$

Saito, Shinichi, 12

Sattari, Hamed, 03

Saurav, Kumar, 03

Scherer, B., 13
Sciancalepore, Corrado, OB

Selvidge, Jennifer G., 04

Sen, Mrinal, $1 \mathrm{U}$

Shang, Chen, 04

Sharif Azadeh, Saeed, OA, OC

Shin, Dongseok, 10

Soref, Richard, 1C

Stirling, Callum J., $1 G$

Stojanovic, Vladimir, OK

Strahl, T., 13

Strain, Michael J., $1 G$

SU, Zhan, OK

Sun, Fei, OG, OV

Szelag, Bertrand, OB, 15

Tahir, W., IP

Takabayashi, Alain Yuji, 03

Talli, Giuseppe, 03

Tappura, Kirsi, 07

Tarazona, Antulio, OY

Teulle, A., 13

Timurdogan, Erman, OK

Tran, Huong, 1C

Ukaegbu, I. A., IK

Vakarin, Vladyslav, OD

Van Campenhout, J., OF

Van, Vien, $1 \mathrm{~J}$

Vehmas, Tapani, OV

Verdot, T., 13

Verheyen, Peter, 03

Virot, L., 15

Vivien, Laurent, OD, 16

Wan, Yating, 04

Wang, Xiaojing, 03

Wang, Yaguo, $\mathrm{OH}$

Wang, Yang, $\mathrm{OH}$

Wang, Yuehai, IR

Watts, Michael R., OK

Weckenmann, Erwan, 16

Wilmart, Quentin, OB, 15

Witzens, Jeremy, OA, OC

Xing, Yufei, 10

$\mathrm{Xu}, \mathrm{Xiaochuan}, \mathrm{OH}$

Yan, Xingzhao, 12

Yang, Jianyi, $1 R$

YU, Hui, IR

Yu, Shui-Qing, $1 C$

Yu, Xingshi, 12

Yun, llgu, 10

Zakwan, M., 1P

Zand, Iman, 03

Zegmout, $\mathrm{H}_{\text {., }} 15$

Zhang, Zeyu, 04

Zhou, Peiji, OP

Zimmerling, Tyler J., $1 \mathrm{~J}$

Zou, Yi, OP 


\section{Conference Committee}

Symposium Chairs

Sailing He, KTH Royal Institute of Technology (Sweden) and Zhejiang University (China)

Yasuhiro Koike, Keio University (Japan)

Symposium Co-chairs

Connie J. Chang-Hasnain, University of California, Berkeley (United States)

Graham T. Reed, Optoelectronics Research Centre, University of Southampton (United Kingdom)

Program Track Chairs

Yakov Sidorin, Quarles \& Brady LLP (United States)

Jean-Emmanuel Broquin, IMEP-LAHC (France)

Conference Chairs

Graham T. Reed, Optoelectronics Research Centre (United Kingdom)

Andrew P. Knights, McMaster University (Canada)

Conference Program Committee

Martijn J. R. Heck, Aarhus University (Denmark)

Siegfried Janz, National Research Council Canada (Canada)

Delphine Marris-Morini, Centre de Nanosciences et de Nanotechnologies (France)

Goran Z. Mashanovich, University of Southampton (United Kingdom) Jurgen Michel, Massachusetts Institute of Technology (United States) Liam O'Faolain, Tyndall National Institute (Ireland)

Jason Ching Eng Png, A*STAR Institute of High Performance Computing (Singapore)

Andrew W. Poon, Hong Kong University of Science and Technology (Hong Kong, China)

Haisheng Rong, Intel Corporation (United States)

Dries Van Thourhout, University Gent (Belgium)

Laurent Vivien, Centre de Nanosciences et de Nanotechnologies (France)

Jeremy Witzens, RWTH Aachen University (Germany)

Winnie N. Ye, Carleton University (Canada)

Shui-Qing Yu, University of Arkansas (United States) 
Zhiping Zhou, Peking University (China)

Aaron J. Zilkie, Rockley Photonics (United States)

\section{Session Chairs}

1 Photonic Integration

Andrew P. Knights, McMaster University (Canada)

2 Optical Communications I

Graham T. Reed, Optoelectronics Research Centre (United Kingdom)

3 Optical Communications II

lain F. Crowe, The University of Manchester (United Kingdom)

4 Silicon Photonics Enabled LIDAR

Andrew P. Knights, McMaster University (Canada)

5 Waveguides

Dan-Xia Xu, National Research Council Canada (Canada)

6 Ge/Si Integration

Dylan F. Logan, RANOVUS, Inc. (Canada)

$7 \quad$ Manufacturing Technology

Iain F. Crowe, The University of Manchester (United Kingdom)

8 Optical Detection and Sensing I

Jens H. Schmid, National Research Council Canada (Canada)

9 Emerging Applications I

Andrew P. Knights, McMaster University (Canada)

10 Emerging Applications II

Jonathan K. Doylend, Intel Corporation (United States)

11 Optical Detection and Sensing II

Graham T. Reed, Optoelectronics Research Centre (United Kingdom)

12 Optical Communications III

Graham T. Reed, Optoelectronics Research Centre (United Kingdom) 